\title{
Point-of-care ultrasound rapidly and reliably diagnoses renal tract obstruction in patients admitted with acute kidney injury
}

\author{
Authors: Samiksha Nepal, ${ }^{A}$ Martin Dachsel ${ }^{B}$ and Nicholas Smallwood ${ }^{A}$
}

Point-of-care ultrasound (POCUS) is increasingly being used in the management of the acutely unwell patient. Its role in the breathless and shocked patient is well established, but less so for those patients with acute kidney injury (AKI). This local quality improvement project looked at the diagnostic accuracy of a protocolised POCUS versus departmental ultrasound. It provides the first data within the internal medicine setting to suggest that POCUS can rapidly and reliably diagnose renal tract obstruction, showing a sensitivity of $90 \%$ and a specificity of $100 \%$. Additionally, negative predictive value was $99 \%$ suggesting it is a tool which can reduce the need for departmental ultrasound. More work is needed to see if this data is generalisable to other internal medicine settings, and to assess potential outcomes on patient management and length of stay.

KEYWORDS: Point-of-care ultrasound, acute kidney injury, hydronephrosis, FAMUS, POCUS

DOI: $10.7861 /$ clinmed.2019-0417

\section{Introduction}

The potential benefits of point-of-care ultrasound (POCUS) are being increasingly recognised. ${ }^{1}$ Advances in technology mean reliable ultrasound machines can be taken to the bedside which, in the right hands, has the potential to reduce the time to diagnosis and increase diagnostic accuracy versus clinical assessment alone. ${ }^{2}$ Despite this, there remains relatively little evidence for the utility of POCUS in the internal medicine setting, and particularly in the assessment of patients with acute kidney injury (AKI). A recent small (30 patient) study conducted in the intensive care unit (ICU) setting evaluated whether physician-delivered POCUS could reliably image the renal tract in patients with AKI; the authors concluded they could. ${ }^{3}$ A further small scale study of emergency physician-delivered ultrasound versus departmental ultrasound included 10 patients with AKI, and showed a $100 \%$

Authors: ${ }^{\text {A }}$ clinical fellow in acute medicine, East Surrey Hospital, Redhill, UK; ${ }^{\mathrm{B}}$ consultant in acute medicine, East Surrey Hospital, Redhill, UK negative predictive value for the absence of hydronephrosis. ${ }^{4}$ To our knowledge, there has not been any previously published data assessing the use of POCUS to aid the management of patients admitted to the hospital outside the emergency department and ICU with an AKI.

AKI is common (it is seen in $13-18 \%$ of patients admitted to hospital) and associated with a significant morbidity and mortality. ${ }^{5}$ Part of the assessment of those diagnosed with an AKI involves determining the risk of outflow tract obstruction, and often therefore a renal tract ultrasound is required to assess for the presence of hydronephrosis or a distended bladder. It was precisely because of the frequency with which AKI is seen in acute medical units (AMUs) that an assessment of the renal tract was included as a core component of the focused acute medicine ultrasound (FAMUS) curriculum. Other components are lung ultrasound for respiratory failure and pleural effusions, localisation of ascites, deep vein thrombosis (DVT) rule-in scans and ultrasound-guided vascular access. ${ }^{6}$ This quality improvement project aimed to assess whether physician-delivered renal tract POCUS according to the FAMUS curriculum could rapidly and reliably assess the renal tract for obstruction in patients admitted to the AMU with an AKI.

\section{Method}

This was a prospective observational study of a convenience sample of patients admitted to a single AMU with various stages of AKI between July 2018 and December 2018. Patients were included when there was a clinician available who was familiar with the FAMUS curriculum. A total of 54 patients were included in the study. The inclusion criteria for patients were documented AKI in adult patients, in whom a renal tract ultrasound was necessary as part of their usual care. Patients who had already had a departmental scan performed were excluded from the study, as this was a quality improvement project to determine the impact of physician-delivered POCUS prior to departmental imaging. No patients were excluded based on potential technical difficulties (body habitus or immobility).

After inclusion, a POCUS was performed by acute physicians according to the FAMUS curriculum. The scanning was performed using a Sonosite Xporte ultrasound machine with a $2-5 \mathrm{MHz}$ curvilinear probe, setup with the 'abdomen' profile. Participating physicians were FAMUS supervisors and trainees. The trainees 
Table 1. Timings of point-of-care ultrasound and reports

\begin{tabular}{|c|c|c|c|c|}
\hline & All PoCUS & Trainee POCUS & Supervisor POCUS & Departmental scans \\
\hline $\begin{array}{l}\text { Time taken for POCUS, hours:minutes, median } \\
\text { (1st quartile, 3rd quartile) }\end{array}$ & $0: 06(0: 05,0: 08)$ & $0: 07(0: 05,0: 10)$ & $0: 06(0: 05,0: 07)$ & $\mathrm{n} / \mathrm{a}$ \\
\hline $\begin{array}{l}\text { Time from scan start to report, hours:minutes, } \\
\text { median (1st quartile, 3rd quartile) }\end{array}$ & $0: 10(0: 07,0: 13)$ & $0: 13(0: 07,0: 19)$ & $0: 10(0: 07,0: 12)$ & $\mathrm{n} / \mathrm{a}$ \\
\hline $\begin{array}{l}\text { Time from initial scan request to report, } \\
\text { hours:minutes, median (1st quartile, } 3 r d \text { quartile) }\end{array}$ & $0: 28(0: 14,1: 51)$ & $0: 23(0: 17,1: 00)$ & $0: 29(0: 12,3: 23)$ & $22: 54(7: 24,29: 37)$ \\
\hline
\end{tabular}

$\mathrm{n} / \mathrm{a}=$ not applicable; POCUS = point-of-care ultrasound .

were physicians who had attended a 1-day FAMUS course and were in the process of acquiring FAMUS accreditation. After every scan, a reporting form was completed which included time taken to perform and report; presence and degree of hydronephrosis; other significant abnormalities; and whether a departmental scan would be considered necessary. Hydronephrosis was defined as dilatation of the renal collecting system and was measured visually as mild, moderate or severe. The clinician performing the scan self-reported if they felt the images were diagnostic.

A departmental scan (renal ultrasound or computed tomography (CT) of the renal tract) was then performed as per the initial management plan, the departmental reporter being blind to the POCUS results. The POCUS scan was used to guide patient management if findings were agreed by a supervisor, but all patients had confirmatory imaging requested. The results were then compared for each patient.

Primary outcomes were to determine the accuracy of POCUS versus departmental imaging, alongside time to scan and report. Secondary outcomes included number of significant pathologies (other than hydronephrosis) reported accurately by POCUS.

Gaussian distributed parameters are shown as mean \pm standard deviation. Non-Gaussian distributed parameters are shown as median (1st quartile, 3rd quartile).

\section{Results}

A total of 54 patients were included in the quality improvement project; $48 \%$ were diagnosed with AKI stage $3,40 \%$ with AKI stage 2 and $12 \%$ with AKI stage 1 . Out of these 54 patients, 36 were scanned by FAMUS supervisors and 18 were scanned by trainees.

Out of the 54 patients, one patient had a single kidney (left nephrectomy), one patient had three kidneys (transplant), and two patients were found to have atrophic kidneys. A total of 106 kidneys were therefore scanned, with 102 (96.2\%) adequately imaged by POCUS. In five patients, no departmental imaging was subsequently performed; this was either due to being cancelled by the team, commenced on end-of-life care or the patient not tolerating the scan. Ninety-six kidneys were therefore used for comparative analysis.

All timings showed a right skewed distribution, so median times and interquartile ranges were used. The median time to perform the POCUS was 6 minutes $(5,8)$. The median time from commencing the scan to the formal POCUS report was 10 minutes $(7,13)$. The median time taken for POCUS report to be generated from the time of the initial scan request was $0: 28$ hours $(0: 14,1: 51)$, whereas the median time for a departmental scan from the initial scan request was 22:54 hours $(7: 24,29: 37)$. All the timings (including the split between trainees and supervisors) are shown in Table 1.

Hydronephrosis was noted in five patients on POCUS (bilateral: 4 patients; unilateral: 1 patient; a total of nine kidneys (8.6\%)), which were all confirmed by formal ultrasound or CT of kidneys, ureters and bladder (specificity $100 \%$; positive predictive value $100 \%$; see Table 2). Mild hydronephrosis was noted in two kidneys and moderate hydronephrosis was noted in seven kidneys.

One kidney was identified as without hydronephrosis on POCUS, but was reported as mild hydronephrosis on departmental CT. The patient did not require any further intervention or management of the hydronephrosis. Hence, nine out of 10 kidneys with hydronephrosis were correctly identified (sensitivity $90 \%$; negative predictive value $99 \%$ ).

One patient with bilateral hydronephrosis secondary to urinary retention on POCUS was immediately catheterised. A departmental scan performed 26 hours later showed a resolution of the hydronephrosis. Both POCUS and radiology images were confirmed by a radiologist for adequacy and accuracy of the findings. For the purpose of result interpretation, this has been included as true positive result.

While a FAMUS point-of-care ultrasound of the kidneys does not look at anything other than hydronephrosis, renal cysts in 17 kidneys and a neobladder in one patient were found. All of these findings were confirmed in the departmental renal scan. There were no significant findings (requiring further investigations) noted in the departmental imaging that were not reported with POCUS imaging.

Table 2. Test statistics for all users (supervisors and trainees)

\section{Hydronephrosis seen in departmental scan}

Hydronephrosis in POCUS

Hydronephrosis not seen in POCUS
9

1

Sensitivity: 90\% (9/10)

\section{Hydronephrosis not seen in departmental scan}

0

86

Specificity: $100 \%(86 / 86)$

NPV = negative predictive value; POCUS = point-of-care ultrasound; PPV = positive predictive value. 


\section{Discussion}

This quality improvement project shows that using POCUS as part of patient management in AKI is quick, accurate and saves time compared with traditional departmental imaging. If this were to be replicated more widely, it could have significant positive implications for patient care (ensuring accurate diagnoses more rapidly), and in reducing the strain on radiology services. This is the first published work looking into the use of POCUS in the internal medicine setting.

Diagnostic images were obtained using POCUS for over $95 \%$ of kidneys in patients with AKI, regardless of frailty, body mass index or patient preparation. The median duration of a POCUS was 6 minutes, suggesting it could be integrated into the standard work of acute physicians and trainees. The average time saved by performing POCUS rather than waiting for departmental imaging was over 22 hours (often over the weekend). This has significant implications for patient care and may possibly also reduce length of stay. It is important to note, however, that this study only included those patients who hadn't already had departmental imaging at the time of enrolment, so 22 hours does not reflect the average time all patients with AKI would save through POCUS, as some would be imaged in the department before a POCUS trained clinician was available. Additionally, POCUS at the point of admission does not necessarily mean that patients will not require a departmental scan so as not to miss other pathology.

Aside from potential time savings, this study highlights that acute physicians can accurately identify hydronephrosis in patients with AKI. POCUS performed at the time of admission by both FAMUS supervisors and trainees showed a good sensitivity $(90 \%)$ and specificity (100\%) for hydronephrosis. We noted high positive $(100 \%)$ and negative $(99 \%)$ predictive values, consistently ruling hydronephrosis in and out. The single false negative case was of mild hydronephrosis which required no specific change in management.

In our study, there were nine kidneys with hydronephrosis (8.6\% of total scans), which reflects the prevalence of post-renal AKI in hospital admissions under the acute medical take (post-renal AKI accounting for $10 \%$ of total hospital admissions with AKI). ${ }^{5}$ One patient was correctly identified as having bilateral hydronephrosis on POCUS and subsequently had urinary catheter placement, causing resolution of renal tract dilatation on departmental scan over 24 hours later.

One strength of this study is that we utilised both POCUS trainees and supervisors to perform the scans, and showed equivalent results for both groups. This suggests the protocolised approach to renal tract imaging described in the FAMUS curriculum is robust in detecting hydronephrosis. It also suggests that these benefits do not require experienced POCUS supervisors, but can also be seen by those who are completing the FAMUS training pathway. This makes the benefits achievable in areas who do not currently have experienced POCUS practitioners, as long as they have clinicians who have completed the accreditation process.

The main drawback of this work is that it was local quality improvement, and so the findings are not necessarily generalisable to other departments or patient populations. This applies particularly to the time savings versus departmental imaging, which will be very dependent on local imaging services. Additionally, given it was quality improvement there was no control group utilised, therefore no inference about the value of the intervention can easily be made. However, it is intuitive to think that accurate, rapid diagnostic tests will allow definitive management to be instigated more quickly, and therefore seems reasonable to infer that POCUS will improve patient care, and possibly help reduce patient length of stay. Certainly, the removal of a trip to the radiology department with all that entails is likely to be viewed positively by patients. We know that, overall, patients have been enthusiastic about the use of POCUS within their care. ${ }^{7,8}$

A similar study done by Parulekar et al looked into performance of POCUS in a smaller group of patients with AKI in the ICU, and also showed accuracy with bedside imaging. ${ }^{3}$ Our study involves a larger sample size with more positive hydronephrosis scans, which is likely due to the fact that all of our patients were scanned at the point of admission prior to catheterisation (as opposed to patients in ICU who were already catheterised prior to POCUS as a part of their resuscitation). A study conducted by Javaudin et al in emergency settings showed that a short training programme enabled emergency physicians to correctly rule out hydronephrosis with POCUS. ${ }^{4}$ Another study done by Caronia et al demonstrated that after a 5 -hour training course, medical residents accurately identified hydronephrosis and key sonographic findings of chronic kidney disease in medical wards and medical intensive care unit. ${ }^{9}$ However, no previous study has focused on the management of patients admitted to internal medicine / AMU with AKI.

POCUS is rapid, easy to perform once learned and findings are immediately available, while formal departmental ultrasound has to be requested, performed and interpreted which results in delays. Hydronephrosis due to obstruction requires immediate intervention, which may be delayed while waiting for a departmental scan; our study shows that in these cases there is potential for a significant reduction in time to definitive management. We show that POCUS has the potential to become an invaluable extension of physical examination, and aids in early diagnosis and management of acutely unwell patients. Currently, POCUS is not a mandatory part of the acute internal medicine or internal medicine curricula, and only a limited number of acute physicians/trainees have been trained. ${ }^{10}$ However, we feel that incorporating POCUS training into internal medicine curricula should be strongly considered, given the potential benefits for improvements in patient management. Further work is also required to see if these local findings are reproducible across other admission units and internal medicine settings, and how it may impact on patient experience and length of stay.

\section{Conflicts of interest}

Martin Dachsel and Nicholas Smallwood are members of FAMUS working group.

\section{References}

1 Laursen CB, Sloth E, Lassen AT et al. Point-of-care ultrasonography in patients admitted with respiratory symptoms: a single-blind, randomised controlled trial. Lancet Respir Med 2014;2:638-46.

2 Koratala A, Segal S, Kazory A. Integrating point-of-care ultrasonography into nephrology fellowship training: a model curriculum. American Journal of Kidney Disease 2019;74:1-5.

3 Parulekar P, Neil-Gallacher E, Harrison A. Intensive care unit physician-delivered point of care renal tract ultrasound in acute kidney injury is feasible. Journal of Intensive Care Society 2018;19;313-8.

4 Javaudin F, Mounier F, Pes P et al. Evaluation of a short formation on the performance of point-of-care renal ultrasound by physicians without previous ultrasound skills. Crit Ultrasound J 2017;9:23. 
5 National Institute for Health and Care Excellence. Acute kidney injury: prevention, detection and management: NICE guideline [NG148]. NICE, 2019.

6 Smallwood N, Dachsel M, Matsa R, Tabiowo E, Walden A. Focused acute medicine ultrasound (FAMUS) - point of care ultrasound for acute medical unit. Acute Med 2016;15:193-6.

7 Howard ZD, Noble VE, Marill KA et al. Bedside ultrasound maximizes patient satisfaction. J Emerg Med 2014;46:46-53.

8 Claret PG, Bobbia X, Le Roux S et al. Point-of-care ultrasonography at the ED maximizes patient confidence in emergency physicians. Am J Emerg Med 2016;34:657-9.

9 Caronia J, Panagopoulos G, Devita M et al. Focused renal sonography performed and interpreted by Internal Medicine Residents. J Ultrasound Med 2013:32:2007-12
10 Smallwood N, Matsa R, Lawrenson P, Messenger J, Walden A. A UK wide survey on attitudes to point of care ultrasound training amongst clinicians working on the Acute Medical Unit. Acute Medicine 2015;14:158-68.

Address for correspondence: Dr Nicholas Smallwood, Acute Medicine Department, East Surrey Hospital, Canada Avenue, Redhill, Surrey RH1 5RH, UK.

Email: nicholas.smallwood@nhs.net

\section{Acute care toolkit 15: Managing acute care problems in pregnancy}

Pregnant women can present to any acute hospital service at any time during their pregnancy or the postpartum period, up to 12 months post-delivery. Women may present with acute medical problems that need to be managed differently because of pregnancy, or may present with obstetric syndromes.

This toolkit provides practical guidance on managing women with acute medical problems in pregnancy. It also advises healthcare staff to take the opportunity to ask about a woman's mental wellbeing when she comes to hospital for other reasons during or after pregnancy; women with a history of mental health problems are more likely to develop new symptoms during pregnancy and postpartum.

Essential reading for front-line NHS staff who may be unfamiliar with the normal physiology of pregnancy and/or diseases that present in pregnancy.

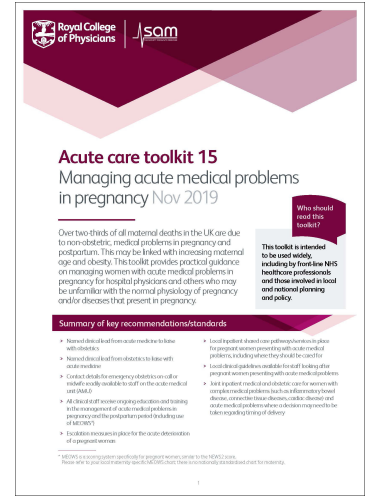

Download now at: www.rcplondon.ac.uk/act15 